\title{
IMPLICAÇÕES ODONTOLÓGICAS DO ACOMETIMENTO PELA DOENÇA CELÍACA
}

DENTAL IMPLICATIONS OF CELIAC DISEASE

Sérgio Spezzia

Cirurgião Dentista e Mestre em Ciências pela Escola Paulista de Medicina Universidade Federal de São Paulo.

\section{Autor Principal:}

Sérgio Spezzia

Email: sergio.spezzia@unifesp.br 


\section{Resumo}

Doença celíaca (DC) consta de desordem inflamatória crônica autoimune, que desencadeia reação autoimune na região do intestino delgado. As modificações ocasionadas pela DC na mucosa do intestino delgado possuem reversibilidade, ao passo que orienta-se os pacientes a não ingerir mais alimentação que contenha o glúten. Deve ser adotada também como medida a averiguação das bulas dos remédios, no intuito de verificar se os mesmos contém traços de glúten em sua fórmula. Existem inúmeros pacientes assintomáticos, o que dificulta a determinação diagnóstica e sabe-se que caso não se proceda ao diagnóstico e ao tratamento da DC em tempo hábil pode haver complicações indesejáveis. O objetivo do presente artigo foi averiguar acerca das manifestações bucais ocorridas em pacientes doentes em decorrência da presença da DC. As principais manifestações orais da DC que podem ocorrer envolvem hipossalivação; glossites; defeitos de esmalte; úlceras aftosas recorrentes; estomatites; aftas; manchas por insuficiência ou por excesso de cálcio, dentre outras. O conhecimento e a conscientização prévia pelos cirurgiões dentistas acerca das repercussões bucais acarretadas pela possível presença da DC em seus pacientes é de fundamental importância para o aprimoramento diagnóstico e para a adoção de condutas odontológicas apropriadas.

Palavras-chave: Doença Celíaca. Dieta. Diagnóstico Precoce. Hipoplasia do Esmalte Dentário.

Keywords: Celiac Disease. Diet. Early Diagnosis. Dental Enamel Hypoplasia. 


\section{INTRODUÇÃO}

Doença celíaca (DC), que pode ser designada como Enteropatia glúten induzida, Enteropatia glúten-sensível, Espru celíaco ou Espru não-tropical consta de doença inflamatória crônica autoimune, que desencadeia reação autoimune na região do intestino delgado, quando determinados indivíduos ingerem o glúten ou proteínas semelhantes originadas do trigo (gliadina), centeio (hordeína), aveia (avenina) e cevada (sevalina). Nos portadores da DC ocorre má absorção dos nutrientes, em decorrência da atrofia que estabelece-se nas vilosidades do intestino delgado. As modificações ocasionadas pela DC na mucosa do intestino delgado possuem reversibilidade, ao passo que orienta-se os pacientes a não ingerir mais alimentação que contenha o glúten (WORLD GASTROENTEROLOGY ORGANIZATION, 2012; CATASSI, C, et al., 2014; LIU, SM, et al., 2014; BRASIL, 2015; GARNIER-LENGLINE, H, et al., 2015; MEIJER, CR, et al., 2015; THEETHIRA, TG \& DENNIS, M, 2015; LEBWOHL, B, et al., 2016; PAUL, SP, et al., 2016; RESENDE, PVG, et al., 2017; CANADÁ, 2018).

Sabe-se que a instalação dessa doença, advém de fatores de ordem ambiental, genética e imunológica e da ingestão de alimentação com glúten como componente. Nela atuam auto-anticorpos, que realizam enfrentamento a presença do glúten por via imunológica (KOTZE, LMS, 1998; GUEVARA, GP, 2002; KOTZE, LMS, 2006; WORLD GASTROENTEROLOGY ORGANIZATION, 2012).

O consumo de glúten não pode ocorrer em pacientes com a DC, uma vez que mesmo ocorrendo o contato com uma infíma quantia de glúten podem instalar-se complicações envolvendo, problemas intestinais, redução de peso, diarreia, dor no abdômen e osteoporose, entre outras (SANTOS, CHS, et al., 2002; NASCIMENTO, KO, et al., 2012; SIMPSON, S \& THOMPSON, T, 2012; CATASSI, C, et al., 2014).

Em âmbito odontológico existe a manifestação clínica de muitos sinais e sintomas da DC na região da cavidade bucal, o que muitas vezes pode levar ao estabelecimento do diagnóstico da doença, uma vez que pode-se conviver apenas com essas manifestações da doença no transcorrer dos atendimentos de saúde (RASHID, M, et al., 2011; BRAMANTI, E, et al., 2014; CANTEKIN, K, et al., 2015; DE CARVALHO, FK, et al., 2015; GUERRA, FA, 2015; CRUZ, ITSA, 2016; DANE, A \& GURBUZ, T, 2016; VAN GILS, T, et al., 2017). 
Uma abordagem preventiva frente a doença é fundamental, visando evitar-se complicações como a ocorrência de suas manifestações bucais. Procedimentos preventivos, envolvendo o tratamento sistêmico da DC mostram-se salutares (GAILLARD, JMPC, 2017).

$\mathrm{O}$ objetivo do presente artigo foi averiguar acerca das manifestações bucais ocorridas em pacientes doentes em decorrência da presença da DC.

\section{REVISÃO DE LITERATURA}

Epidemiologicamente, sabe-se que a prevalência apresentada pela DC demonstra variabilidade entre os países, instalando-se comumente na Europa (LANDABURO, RV \& PEREZ, FS, 2002; VAN DER WINDT, DAWM, 2010).

A DC pode ser classificada em assintomática e sintomática. $\mathrm{Na}$ forma sintomática existe presença de sintomatologia gastrointestinal clássica, englobando, palidez, distensão abdominal, flatulência, vômito, diarreia crônica, fadiga, anorexia, redução do peso, má absorção, entre outras manifestações clínicas, já na forma assintomática não existe presença dessa sintomatologia considerada clássica e são levados em conta outros sinais para determinação diagnóstica (GREEN, PH \& JABRI, B, 2006; NASCIMENTO, KO, et al., 2012; WORLD GASTROENTEROLOGY ORGANIZATION, 2012; BOZZOLA, M, et al., 2014; BRASIL, 2015).

Existem outras classificações da DC, dentre elas uma que se encontra em conformidade com as formas clínicas que são evidenciadas pela doença, constituindo, DC típica ou clássica; DC atípica; DC silenciosa ou assintomática; DC latente e DC refratária (NIH, 2004; CUPERTINO, LH, et al., 2016).

O diagnóstico da DC é firmado com a realização de anamnese minuciosa, exame físico, averiguação e análise histopatológica do intestino delgado, empregando-se testes sorológicos de anticorpos específicos, tais como: antiendomisial, antigliadina e anticorpos de antitransglutaminase, entretanto, a elaboração do diagnóstico final deve levar em consideração o resultado da biópsia intestinal que foi realizada. Muitos pacientes possuidores da DC somente são diagnosticados tardiamente, constituindo um problema. Em contrapartida seria conveniente que o diagnóstico fosse firmado precocemente para evitar-se complicações (WETIZ, JCV, et al., 2003; AGA, 2006; 
ROSTOM, A et al., 2006; CATALDO, F \& MONTALTO, G, 2007; DE SOUZA, AJL, 2010; RICHA, W, et al., 2015; PAUL, SP, et al., 2016; RASHID, M \& LEE, J, 2016; JORGE, AFR, 2018).

Existem inúmeros pacientes assintomáticos, o que dificulta a determinação diagnóstica e sabe-se que caso não se proceda ao diagnóstico e ao tratamento da $\mathrm{DC}$ em tempo hábil pode haver complicações indesejáveis, que poderiam ser evitadas. O conhecimento aprimorado da DC pelo cirurgião dentista pode ajudar na elaboração do diagnóstico (GUEVARA, GP, 2002; GOTTELAND, RM, et al., 2003; CATALDO, F \& MONTALTO, G, 2007; PAUL, SP, et al., 2016; RASHID, M \& LEE, J, 2016; DE CASTRO, AM, 2018).

O tratamento preconizado que possui resolutividade e possibilita remissão da sintomatologia inerente a DC, consta da retirada de alimentos que contém glúten das refeições. Os medicamentos prescritos aos celíacos devem receber avaliação criteriosa ao passo que o glúten pode fazer parte como excipiente de comprimidos, cápsulas e de suspensões orais, o que repercutiria desfavoravelmente, caso os fármacos com glúten na composição fossem prescritos a esses doentes, portanto, é imprescindível que os próprios pacientes tenham consciência de que não podem fazer uso de medicamentos nessas condições. Deve ser adotada como medida a averiguação das bulas dos remédios, no intuito de verificar se os mesmos contém traços de glúten em sua fórmula (OLSON, GB \& GALLO, GR, 1983; ROESSLER, JL, et al., 2001; SDEPANIAN, VL, et al., 2001; RAUEN, MS, et al., 2005; DE OLIVEIRA, TWN, et al., 2018).

A DC possui manifestações sistêmicas e bucais. As principais manifestações orais da DC que podem ocorrer envolvem hipossalivação; glossites; defeitos de esmalte; úlceras aftosas recorrentes; estomatites; aftas; manchas por insuficiência ou por excesso de cálcio, dentre outras. Em particular, existem determinadas situações em que a DC pode acarretar desnutrição proteico calórica, o que pode levar a instalação de problemas bucais, como: atraso na erupção dentária; disfunção nas glândulas salivares e redução do tamanho dos dentes (RASHID, M, et al., 2011; BRAMANTI, E, et al., 2014; CANTEKIN, K, et al., 2015; DE CARVALHO, FK, et al., 2015; GUERRA, FA, 2015; CRUZ, ITSA, 2016; DANE, A \& GURBUZ, T, 2016; LOPES, SLD, 2016; GONÇALVES, JBS, 2017; VAN GILS, T, et al., 2017). 
Em crianças nos seus dois primeiros anos, pode-se conviver com a doença, que desencadeia manifestações sistêmicas e orais, como: crescimento deficitário; vômitos; diarreia; redução no fluxo salivar; defeitos de esmalte, que constituem uma das mais importantes manifestações da DC na cavidade bucal e ulcerações aftosas recorrentes. Hipoplasia do esmalte dentário pode constituir sinal da presença da DC, ocorre que em crianças e adolescentes, por vezes a hipoplasia pode ser o único indício presente da doença (AINE, L, 1994; AINE, L, 1996; RASHID, M, et al., 2011; GOMEZ, TPM, et al., 2012; DE CASTRO, AM, 2018).

Relacionado ao atraso provocado na erupção dentária, nos portadores de DC, em decorrência da doença, ocorre insuficiente aproveitamento dos elementos nutritivos da dieta, o que ocasiona prejuízo no crescimento e desenvolvimento concomitantes. O desenvolvimento dentário nessas circunstâncias pode ser afligido, devido período extenso da prática de má absorção, ocorrendo atraso significativo na erupção (FERRAZ, EG, et al., 2012; KRZYWICKA, B, 2014).

\section{DISCUSSÃO}

A lei no. 10.674 instituída no ano de 2003 em nosso país, formalizou medida que tornava obrigatória menção nos gêneros de alimentação, informando quando os mesmos continham ou não glúten, visando preventivamente evitar-se a instalação de danos nos indivíduos que possuíam a DC (BRASIL, 2003).

Segundo estudo realizado no ano de 2014, o acometimento pela DC tornou-se mais elevado nos últimos anos. Europa e Estados Unidos constituem países em que são consumidos muitos produtos alimentícios com glúten incorporado, devido ao fato essas localidades possuem prevalência aumentada de DC (CATASSI, C, et al., 2014).

$\mathrm{Na}$ literatura, no contexto geral, existe menção evidente do relacionamento existente entre DC e a presença dos defeitos de esmalte nas populações de diferentes localidades (GONÇALVES, JBS, 2017).

Evidenciou-se em alguns estudos que existe correlação entre DC e alterações bucais, envolvendo defeitos do esmalte, redução do fluxo salivar e estomatite aftosa recorrente (GONÇALVES, JBS, 2017). 
Estudos afirmam que deve-se averiguar acerca das manifestações orais presentes nos pacientes, procurando possível correlação com a DC, o que pode facilitar e tornar precoce o diagnóstico da DC (BOZZOLA, M, et al., 2014; DE CARVALHO, FK, et al., 2015).

Estudo de revisão bibliográfica acerca da DC preconizado por KOTZE, (1998), identificou como manifestações bucais provenientes da DC, a glossite, a língua despapilada e a queilite angular.

Estudo realizado examinando-se 128 pacientes portadores de DC e usuários de alimentação isentada do glúten verificou a presença de manifestações orais da doença, examinou-se também 30 pacientes como controle. No desenvolvimento desse estudo avaliou-se a história médica dos participantes, verificando prováveis patologias que poderiam estar relacionadas a DC e realizou-se exame clínico, visando identificação das repercussões orais da DC. Resultados obtidos denotaram presença de manifestações bucais, advindo da DC, tais como: ardência lingual, eritema e ulceração em língua, lábios e palato. Úlceras foram as lesões bucais presentes com maior frequência (LAHTEENOJA, H, et al., 1998).

Estudos realizados nos Estados Unidos na década de 1990 e que averiguaram 59 compostos medicamentosos detectaram gliadina em $71 \%$ deles. Outro estudo efetuado na Iugoslávia nesse mesmo período e que verificou a composição de 47 medicamentos detectou gliadina em 31\% (SDEPANIAN, VL, et al., 2001).

No Brasil realizou-se estudo similar, visando identificação de gliadina em medicamentos disponíveis para utilização dos pacientes, como antidepressivos, antiácidos, antibióticos e anticonvulsivantes, dentre os 78 medicamentos estudados, somente verificou-se gliadina em cerca de $1 \%$, inexistindo dosagem que causasse problemas a indivíduos usuários com DC (SDEPANIAN, VL, et al., 2001).

O prognóstico e tratamento precoces da DC fornece ao doente melhor qualidade de vida, evitando a ocorrência de complicações oncológicas, como neoplasias gastrointestinais, que detém maior gravidade. O cirurgião dentista deve conhecer a doença e proceder, possibilitando diagnóstico e prognóstico aprimorado da DC (BRANDT, KG \& SILVA, GAP, 2003).

Estudo prospectivo realizado por CAMPISI et al., (2007), verificou atraso na erupção dentária ocasionado pela DC. Nesse estudo avaliou-se 107 crianças portadoras 
de DC, destas 28 ou cerca de $27 \%$ do total possuía atraso na erupção dentária. No grupo controle empregou-se 233 crianças e identificou-se somente 16 casos ou cerca de $7 \%$ do total de atraso na erupção dentária.

\section{CONCLUSÃO}

O conhecimento e a conscientização prévia pelos cirurgiões dentistas acerca das repercussões bucais acarretadas pela possível presença da DC em seus pacientes é de fundamental importância para o aprimoramento diagnóstico e para a adoção de condutas odontológicas apropriadas.

\section{REFERÊNCIAS}

1. World Gastroenterology Organization Global Guidelines. Doença celíaca, 2012. Disponível em: http://www.worldgastroenterology.org/UserFiles/file/guidelines/celiacdisease-portuguese-2012.pdf Acessado em 18 de julho de 2019.

2. Catassi C, Gatti S, Fasano A. The New Epidemiology of Celiac Disease. J Pediatr Gastroenterol Nutr, 2014; 59(1):S7-S9.

3. Liu SM, Resende PVG, Bahia M, Penna FJ, Ferreira AR, Liu PMF, et al. Doença Celíaca. Rev Med Minas Gerais, 2014; 24(2):38-45.

4. Brasil. Ministério da Saúde. Protocolo Clínico e Diretrizes Terapêuticas. Doença Celíaca. Portaria SAS/MS no 1149, de 11 de novembro de 2015. Disponível em: http://portalarquivos.saude.gov.br/images/pdf/2016/fevereiro/05/Doen--a-Cel--aca--PCDT-Formatado---port1449-2015.pdf Acessado em 18 de julho de 2019.

5. Garnier-Lengliné H, Cerf-Bensussan N, Ruemmele FM. Celiac disease in children. Clin Res Hepatol Gastroenterol, 2015; 39(5):544-51.

6. Meijer CR, Shamir R, Mearin ML. Coeliac disease and noncoeliac gluten sensitivity. J Pediatr Gastroenterol Nutr, 2015; 60(4):429-3.

7. Theethira TG, Dennis M. Celiac disease and the glutenfree diet: consequences and recommendations for improvement. Digestive Diseases, Basel, 2015; 33(2):175-82. 
8. Lebwohl B, Murray JA, Verdu EF, Crowe SE, Dennis M, Fasano A, et al. Gluten Introduction, Breastfeeding, and Celiac Disease: Back to the Drawing Board. Am J Gastroenterol, 2016; 111:12-4.

9. Paul SP, Khirkhan EN, John R, Stanes K, Basude D. Coeliac disease in children - an update for general dental practitioners. British Dent J, 2016; 220(9):481-5.

10. Resende PVG, Silva NLM, Schettino GCM, Liu PMF. Doenças relacionadas ao glúten. Rev Med Minas Gerais, 2017; 27(Supl 3):S51-S58.

11. Canadá. Managing Food Allergies And Anaphylaxis. A guide for Post-Secondary Institutions, 2018.

Disponível em:

http://foodallergycanada.ca/wpcontent/uploads/Managing-Food-Allergies-andAnaphylaxis.pdf >. Acessado em 24 de novembro de 2018.

12. Kotze LMS. Distúrbios entéricos da absorção. In: Dani R. Gastroenterologia essencial. Rio de Janeiro: Guanabara Koogan; 1998. p. 211-24.

13. Guevara GP. Enfermedad celíaca. Rev Chil Pediatr, 2002; 73(4):394-7.

14. Kotze LMS. Doença Celíaca. J Bras Gastroenterol, 2006; 6:23-34.

15. Santos CHS, Almeida ILB, Gomes MDC, Serafim A, Pereira MM, Muszkat M, et al. Calcificação intracraniana occipital bilateral, epilepsia e doença celíaca. Arq NeuroPsiquiatr, 2002; 60(3-B):840-3.

16. Nascimento KO, Barbosa MIMJ, Takeiti CY. Doença Celíaca: Sintomas, Diagnóstico e Tratamento Nutricional. Saúde Rev, 2012; 12(30):53-63.

17. Simpson S, Thompson T. Nutrition Assessment in Celiac Disease. Gastrointest Endosc Clin North America, 2012; 22:797-809.

18. Rashid M, Zarkadas M, Anca A, Limeback H. Oral manifestations of celiac disease: a clinical guide for dentists. J Mich Dent Assoc, 2011; 93(10):42-6.

19. Bramanti E, Cicciu M, Matacena G, Costa S, Magazzu G. Clinical evaluation of specific oral manifestations in pediatric patients with ascertained versus potential coeliac disease: a cross-sectional study. Gastroentero Res Pract, 2014; Article ID 934159. http://dx.doi.org/10.1155/2014/934159

20. Cantekin K, Arslan D, Delikan E. Presença e distribuição de defeitos do esmalte dentário, lesões aftosas recorrentes e cárie dentária em crianças com doença celíaca. Pak J Med Sci, 2015; 31(3):606-9. 
21. de Carvalho FK, de Queiroz AM, da Silva RAB, Sawamura R, Bachmann L, da Silva LAB, et al. Oral aspects in celiac disease children: clinical and dental enamel chemical evaluation. Oral Surg Oral Med Oral Pathol Oral Radiol, 2015; 119(6):636-43. 22. Guerra FA. Manifestações orais da doença celíaca - Revista da Literatura. Rev Odontologia (ATO), 2015; 15(2):117-49.

23. Cruz ITSA. Manifestações orais em pacientes com doença celíaca. Programa de Pós-graduação em Odontologia. [Dissertação]. Curitiba: Universidade Federal do Paraná, 2016.

24. Dane A, Gurbuz T. Clinical evaluation of specific oral and salivary findings of coeliac disease in eastern Turkish paediatric patients. Eur J Paediatr Dent, 2016; 17(1):53-6.

25. van Gils T, Brand HS, de Boer NKH, Mulder CJJ, Bouma G. Gastrointestinal diseases and their oro-dental manifestations: Part 3: Coeliac disease. Br Dent J, 2017; 222(2):126-9.

26. Gaillard JMPC. Manifestações orais da doença celíaca no paciente pediátrico. [Dissertação]. Porto: Faculdade de Ciências da Saúde - Universidade Fernando Pessoa, 2017.

27. Landaburo RV, Perez FS. Celiaquía: nuevos rostros de una antigua enfermedad. Medicentro, 2002; 6(2):1-7.

28. van der Windt DAWM, Jellema P, Mulder CJ. Diagnostic testing for celiac disease among patients with abdominal symptoms - A Systematic Review. JAMA, 2010; 303(17):1738-46.

29. Green PH, Jabri B. Celiac disease. Annu Rev Med, 2006; 57:207-21.

30. Bozzola M, Bozzola E, Pagani S, Mascolo A, Porto R, Meazza C. Late diagnosis of celiac disease in an asymptomatic infant with growth failure. Riv Ital Pediatr, 2014; 40(1):4.

31. NIH Consensus Development Program- NIH consensus development conference on celiac disease. 2004; 1-16.

32. Cupertino LH, de Oliveira JT, Mucuta PO, Bacelar Júnior AJ, Bacelar LFF. Doença celíaca: uma revisão sistemática. Braz J Surg Clin Res, 2016; 16(3):90-6. 
33. Wetiz JCV, Montalva RD, Alarcon TO, Contreras LM. Determinación de anticuerpos anti-transglutaminasa en el diagnóstico de enfermedad celíaca. Rev Med Chile, 2003; 131(1):25-9.

34. AGA Institute Medical Position Statement on the Diagnosis and Management of Celiac Disease. Gastroenterol, 2006; 131(6):1977-80.

35. Rostom A, Murray JA, Kagnoff MF. American Gastroenterological Association (AGA) Institute technical review on the diagnosis and management of celiac disease. Gastroenterol, 2006; 131(6):1981-2002.

36. Cataldo F, Montalto G. Celiac disease in the developing countries: a new and challenging public health problem. World J Gastroenterol, 2007; 13(15):2153-9.

37. de Souza AJL. Diagnóstico Laboratorial e Aspectos Imunológicos da Doença Celíaca: uma revisão da literatura. [Trabalho de Conclusão]. Brasília: Universidade Católica de Brasília, 2010.

38. Richa W, Yehoshuva R, Puneet RSK, Nitin K, Gaurav S. Oral Manifestations of gastrointestinal disease, an indicator for early diagnosis: an overview. Int J Biopharm, $2015 ; 6(1): 5-12$.

39. Rashid M, Lee J. Serologic testing in celiac disease: Practical guide for clinicians. Can Family Phys, 2016; 62(1):38-43.

40. Jorge AFR. Diagnóstico de doença celíaca através da saliva. [Dissertação]. Porto: Faculdade de Ciências da Saúde - Universidade Fernando Pessoa, 2018.

41. Gotteland RM, Verbeke OS, Cruchet MS. Anticuerpos antitransglutaminasa tissular: una major prueba para detectar trangressiones menores de la dieta em pacientes celiacos? Rev Med Chile, 2003; 131:25-9.

42. de Castro AM. A Relação da Doença Celíaca e a Hipoplasia do Esmalte Dentário. [Trabalho de Conclusão]. Porto Velho: Centro Universitário São Lucas, 2018.

43. Olson GB, Gallo GR. Gluten in pharmaceutical and nutritional products. Am J Hosp Pharm, 1983; 40:121-2.

44. Roessler JL, Ríos GM, Alarcon TO, Bergenfreid CO, Moudragón AO, Araya MQ. Enfermedad celíaca en el adolescente y adulto joven. Un desafío para gastroenterólogos de niños y adultos. Rev Med Chile, 2001; 129(7):743-8. 
45. Sdepanian VL, Scaletsky ICA, Morais MB, Neto UF. Pesquisa de gliadina em medicamentos - informação relevante para a orientação de pacientes com doença celíaca. Arq Gastroenterol, 2001; 38(3):176-82.

46. Rauen MS, Back JCV, Moreira EAM. Doença celíaca: sua relação com a saúde bucal. Rev Nutr, 2005; 18(2):271-6.

47. de Oliveira TWN, Damasceno ANC, Leal LMS, de Sousa RR, Silva CEO, da Silva FE, et al. Dificuldades encontradas pelos pacientes celíacos em seguir a dieta isenta de glúten. Braz J Surg Clin Res, 2018; 24(3):110-5.

48. Lopes SLD. Manifestações Orais da Doença Celíaca. [Dissertação]. Portugal: Instituto Superior de Ciências da Saúde Egaz Moniz, 2016.

49. Gonçalves JBS. Defeitos de desenvolvimento de esmalte como manifestação bucal em pacientes portadores de doença celíaca: revisão de literatura e relato de caso. [Trabalho de Conclusão de Curso]. Brasília: Faculdade de Ciências da Saúde Universidade de Brasília, 2017.

50. Aine L. Permanent tooth dental enamel defects leading to the diagnosis of coeliac disease. Br Dent J, 1994; 177(7):254-5.

51. Aine L. Coeliac-type permanent-tooth enamel defects. Ann Med, 1996; 28(1):9-12.

52. Gomez TPM, Jimeno FG, Dalmau LJB, Tarrida LG. Prevalence of molar-incisor hypomineralisation observed using transillumination in a group of children from Barcelona (Spain). Int J Paediatr Dent, 2012; 22:100-9.

53. Ferraz EG, Campos EJ, Sarmento VA, Rodrigues LS. The oral manifestations of celiac disease: information for the pediatric dentistry. Pediatr Dent, 2012; 34(7):485-8.

54. Krzywicka B. Celiac disease and its impact on the oral health status - Review of the literature. Adv Clin Experim Med, 2014; 23(5):675-81.

55. Brasil. Presidência da República. Lei ${ }^{\circ}$ 10.674, de 16 de maio de 2003. Obriga a que os produtos alimentícios comercializados informem sobre a presença de glúten, como medida preventiva e de controle da doença celíaca. Disponível em:<http://www2.camara.leg.br/legin/fed/lei/2003/lei10674-16-maio-2003-96699normaatualizada-pl.pdf>. Acessado em 10 de novembro de 2018.

56. Lahteenoja H, Toivaner A, Viander M, Maki M, Irjala K, Raiha I, et al. Oral mucosal changes in coeliac patients on a gluten-free diet. Eur J Oral Sci, 1998; 106(5):899-906. 
57. Brandt KG, Silva GAP. Doença Celíaca. Gastroenterologia e Hepatologia em Pediatria: Diagnóstico e Tratamento. Rio de Janeiro, Ed. Medsi, p. 161-74, 2003.

58. Campisi G, di Liberto C, Iacono G, Compilato D, di Prima L, Calvino F, et al. Oral pathology in untreated coeliac disease. Alimentary Pharmacology \& Therapeutics, 2007; 27(6):1529-36. 